\title{
INFLUENCIA DE LA TEMPERATURA DEL AGUA EN LA DISTRIBUCIÓN DE ESTADOS INMADUROS DE SIMULIIDAE (DIPTERA, INSECTA) EN EL RÍO LLUTA, ARICA, CHILE
}

\author{
INFLUENCE OF THE TEMPERATURE OF THE WATER \\ IN THE DISTRIBUTION OF IMMATURE STAGES OF SIMULIIDAE \\ (DIPTERA, INSECTA) IN LLUTA RIVER, ARICA, CHILE
}

Abel A. Henry ${ }^{1}$; Christian R. González ${ }^{2}$

\section{RESUMEN}

Se estudió la relación de la temperatura del agua con la distribución de las especies presentes en el área. Las observaciones fueron realizadas en tres secciones del río Lluta, donde se registraron las temperaturas a las 7.00 AM y 7.00 PM y se colectaron larvas y pupas en sustratos naturales como piedras y vegetación sumergida, procediendo posteriormente a su identificación taxonómica. Los datos obtenidos se analizaron estadísticamente utilizando el coeficiente de correlación de Pearson entre la temperatura media del agua y la cantidad de individuos colectados de cada especie. Las correlaciones obtenidas fueron en S. escomeli de $\mathrm{r}=0,774$, S. philippii de $\mathrm{r}=-0,442$ y $S$. quechuanum $\mathrm{r}=-0,142$. Durante el período de observación la especie $S$. escomeli presentó una correlación positiva intensa respecto a la temperatura del agua,

Palabras clave: Simulium escomeli, S. philippii, S. quechuanum, análisis de correlación, temperatura, río Lluta.

\section{ABSTRACT}

The relation of the temperature of the water with the distribution of the present species in the area was studied.. The observations were made in three sections of the Lluta River, where they registered the temperature to 7:00 A.M. and 7:00 P.M. and collected larvae and pupae in natural substrates like stones and submerged vegetation, coming later to its taxonomic identification. The collected data were analyzed statistically using the coefficient of correlation of Pearson between the average temperature of the water and the amount of individuals collected of each species. The obtained correlations were in S. escomeli of $r=0.774$, S. philippii of $r=-0.442$ and $\mathrm{S}$. quechuanum $r=-0.142$. During the period of observation $\mathrm{S}$. lescomeli presented intense positive correlation with respect to the temperature of the water.

Key words: Simuliidae, Simulium escomeli, S. philippii, S. quechuanum, analysis of correlation, temperature, Lluta river.

\section{INTRODUCCIÓN}

Vannote y Sweeney (1980), estudiando una comunidad acuática, plantean que un régimen térmico óptimo existe para cada especie, maximizando el tamaño del adulto y la fecundidad. Adler y Mc Creadie (1997) relacionan la temperatura como uno de los factores que influye en la macrodistribución de Simuliidae. Lake y Burger (1983) en un área de New Hampshire, discriminan tres grupos de especies de simúlidos de acuerdo a los rangos de temperatura en que fueron colectados, entre $7^{\circ}-10^{\circ} \mathrm{C}$, entre $15^{\circ}$ y $17^{\circ} \mathrm{C}$ y sobre $\operatorname{los} 21^{\circ} \mathrm{C}$. Ross y Merritt (1987) encuentran que larvas invernantes de Prosimulium mixtum son estenotermas

1 Servicio Agrícola y Ganadero, 18 de Septiembre 370, Arica, Chile. Centro de Formación Técnica Santo Tomás, 18 de Septiembre 1231, Arica, Chile. E-mail: abelhenryg2004@yahoo.es

2 Instituto de Entomología, Universidad Metropolitana de Ciencias de la Educación, Casilla 147, Santiago, Chile. E-mail: cgonza@umce.cl

Fecha de Recepción: 14 Julio 2008

Fecha de Aceptación: 07 Noviembre 2008 
de aguas frías y que una temperatura extrema puede gatillar la pupación sincrónica en la especie. Mc Creadie y Colbo (1991, 1992), estudiando el complejo Simulium venustum/verecundum, encuentran una fuerte correlación entre una especie y temperaturas bajas y que también aumenta cuando la temperatura aumenta y la conductividad, oxígeno y el $\mathrm{pH}$ disminuyen. Hadi y Takaoka (1995) estudiaron el efecto de la temperatura sobre el desarrollo de los estados preimaginales de simúlidos encontrando rangos óptimos para las diferentes especies encontradas. Mc Creadie y Adler (1998) predicen la ocurrencia de especies de esta familia de dípteros sobre la base de la temperatura del agua como uno de los factores. Céréghino y Lavandier (1997) asocian la temperatura como uno de los principales factores medioambientales en la distribución y desarrollo larvario en un área donde está ubicada una represa. Gil et al. (1998) estudiando S. wolffhuegeli, S. rubiginosum y $S$. lahillei encuentran que tienen una correlación negativa respecto a este factor. Coscarón et al. (2000) observan la especie $S$. orbitale con correlación negativa respecto a este factor.

El efecto de la temperatura también puede ser indirecto, como lo señalan Nadeau et al. (1995), quienes estudiaron la acción de la temperatura del agua sobre un hongo parásito de larvas de simúlidos que afecta posteriormente las poblaciones adultas.

La problemática central que se propone estudiar en este trabajo es la influencia de un factor abiótico como es la temperatura del agua en la distribución y abundancia de estados inmaduros de Simuliidae en tres secciones del río Lluta seleccionadas en un transecto altitudinal de su cauce, donde presentan una gradiente termal.

\section{MATERIALES Y MÉTODOS}

\section{ÁREA DE ESTUDIO}

El área de estudio escogido fue río Lluta; ubicado en el valle del mismo nombre provincia de Arica presenta un caudal permanente con un promedio de dos metros cúbicos por segundo, nace en la vertiente oriental del volcán Tacora y su recorrido es de 150 kilómetros hasta llegar al mar. Se seleccionaron tres secciones de muestreo del río Lluta que presentaran una gradiente de altitud, lo que debiera reflejar una gradiente termal tanto en la temperatura máxima como mínima:
Sección 1, El Porvenir, Valle de Lluta km 4, $145 \mathrm{msnm}$

Sección 2, Las Chilcas, Valle de Lluta km 20, $540 \mathrm{msnm}$

Sección 3, Tocontasi, Valle de Lluta km 60, $1010 \mathrm{msnm}$

\section{ESTUDIO DE CAMPO}

Se hicieron dos misiones de colectas en cada una de las secciones de muestreo. En cada sección de muestreo se midió la temperatura máxima y mínima del agua y se colectó en una extensión de $10 \mathrm{~m}$ del curso de agua en la vegetación acuática y piedras sumergidas durante 30 minutos, que son los sustratos naturales donde se fijan las larvas y pupas. Las larvas fueron fijadas en alcohol $80^{\circ}$, para posterior estudio; las pupas fueron puestas en frascos con agua corriente para ser criadas posteriormente en laboratorio y obtener imagos para su identificación taxonómica.

\section{ANÁLISIS ESTADÍSTICO}

Los datos utilizados de cada muestra fueron obtenidos de la suma de larvas y pupas de cada especie obtenida de cada sección de muestreo. Se calculó el índice de correlación de Pearson para poder establecer la correlación entre la temperatura media del agua de cada sección del río establecida para el estudio y la presencia de especies, el coeficiente de determinación y la prueba de $t$ (Spiegel 1997).

\section{RESULTADOS}

\section{TEMPERATURA DEL AGUA}

Las temperaturas obtenidas en las tres secciones del río escogidas fueron las siguientes:

El Porvenir: temperatura máxima $24^{\circ} \mathrm{C}$, temperatura mínima $16{ }^{\circ} \mathrm{C}$.

Las Chilcas: temperatura máxima $22^{\circ} \mathrm{C}$, temperatura mínima $15^{\circ} \mathrm{C}$.

Tocontasi: temperatura máxima $21^{\circ} \mathrm{C}$, temperatura mínima $13{ }^{\circ} \mathrm{C}$

La temperatura media del agua en la sección del río analizada presentó una gradiente termal durante el periodo de tiempo de muestreo que fluctuó entre $14,5^{\circ} \mathrm{C}$ y $22,5^{\circ} \mathrm{C}$. 
El rango entre la temperatura mínima y la temperatura máxima fue de $13{ }^{\circ} \mathrm{C}$ y $24^{\circ} \mathrm{C}$.

\section{Presencia de especies}

Se colectaron tres especies en el área de estudio: Simulium (Psilopelmia) escomeli Roubaud 1909, Simulium (Pternaspatha) philippii Coscarón 1976 y S. al( $P$ ) quechuanum Coscarón y Wygodzinsky 1972.
ANÁLISIS DE LOS DATOS:

\section{CORRELACIÓN ENTRE ABUNDANCIA DE CADA ESPECIE ENCONTRADA Y LA TEMPERATURA DEL RÍO}

\section{Cálculo de $\mathbf{r}$}

El coeficiente de correlación obtenido para $S$. (Ps) escomeli Roubaud fue $\boldsymbol{r}=0,774$, para $S$. $(P)$ philippii Coscarón $\boldsymbol{r}=-0,472$ y para $S$. $(P)$ quechuanum Coscarón y Wygodzinsky $\boldsymbol{r}=-142$.

Cuadro 1

Presencia y abundancia de especies de Simuliidae considerando la temperatura media del agua

\begin{tabular}{|c|c|c|c|c|c|c|c|c|c|}
\hline $\mathbf{T}^{\mathbf{0}}$ Celsius & \multicolumn{3}{|c|}{ S. escomeli } & \multicolumn{3}{c|}{ S. philippii } & \multicolumn{3}{c|}{ S. quechuanum } \\
\hline Promedio & $\mathbf{N}^{\mathbf{0}}$ larvas & $\mathbf{N}^{\mathbf{0}}$ pupas & Total & $\mathbf{N}^{\mathbf{0}}$ larvas & $\mathbf{N}^{\mathbf{0}}$ pupas & Total & $\mathbf{N}^{\mathbf{0}}$ larvas & $\mathbf{N}^{\mathbf{0}}$ pupas & Total \\
\hline 14.50 & 0 & 0 & 0 & 11 & 5 & 16 & 4 & 0 & 4 \\
\hline 16.75 & 4 & 0 & 4 & 0 & 0 & 0 & 0 & 0 & 0 \\
\hline 19.00 & 0 & 0 & 0 & 16 & 1 & 17 & 10 & 7 & 17 \\
\hline 19.50 & 17 & 11 & 28 & 0 & 0 & 0 & 0 & 0 & 0 \\
\hline 20.50 & 112 & 6 & 118 & 0 & 0 & 0 & 0 & 0 & 0 \\
\hline 22.50 & 338 & 14 & 352 & 0 & 0 & 0 & 0 & 0 & 0 \\
\hline Totales & 471 & 31 & 502 & 27 & 6 & 33 & 14 & 7 & 21 \\
\hline
\end{tabular}

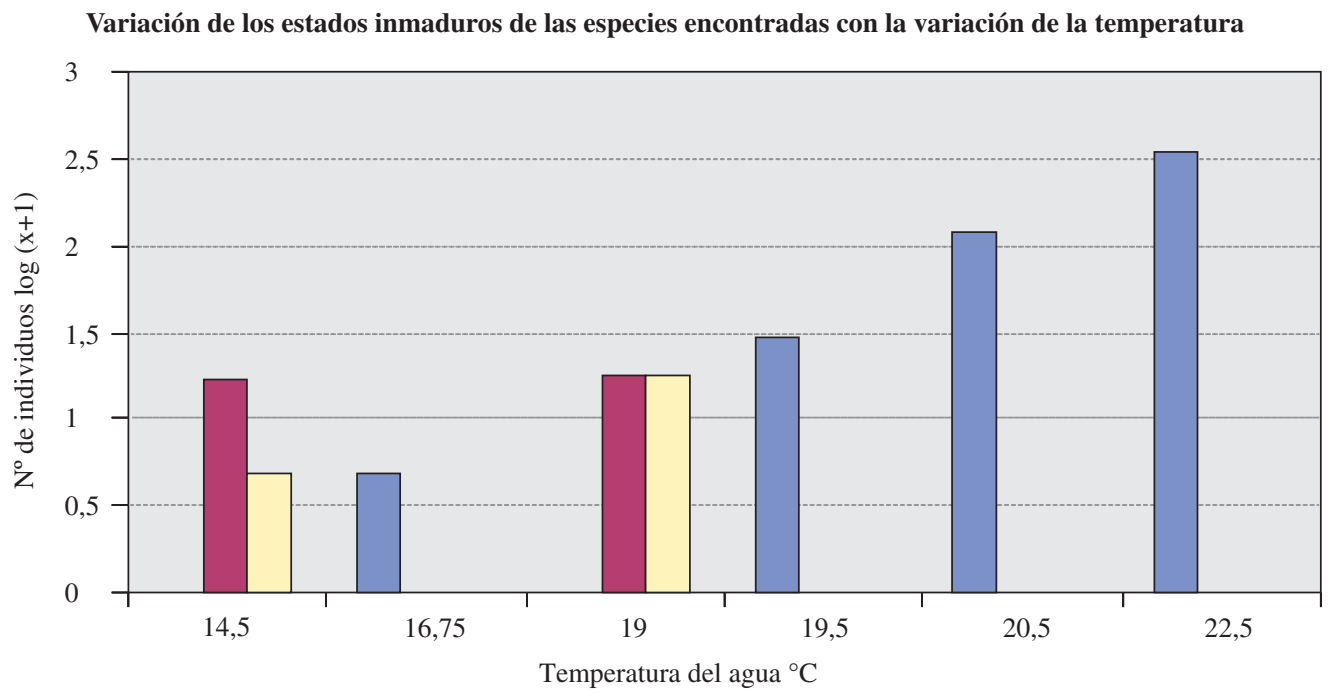

S. escomeli $\square$ S. philippii $\square$ S. quechuanum 


\section{Cálculo de Coeficiente de determinación $\left(r^{2}\right)$}

El coeficiente de determinación obtenido para $S$. (Ps) escomeli Roubaud fue $\boldsymbol{r}^{2}=0,599$, para $S$. $(P)$ philippii Coscarón $\boldsymbol{r}^{2}=0,222$, y para $S$. $(P)$ quechuanum Coscarón y Wygodzinsky $\boldsymbol{r}^{2}=0,020$.

\section{Prueba de significancia de los r calculados}

Usando el nivel de significancia de 5\% $(0,05)$, el valor de tabla es de 2,132. Los resultados obtenidos fueron:

$\boldsymbol{t} S$. escomeli $=2,445$, es decir, existe correlación positiva entre la variación de la temperatura y los ejemplares colectados. Esa correlación no se debe al azar.

$\boldsymbol{t} S$. philippii $=-1,074$, este valor está en el área de aceptación. Por lo tanto no existe correlación negativa entre la variación de la temperatura y los ejemplares colectados. Esa correlación se debe al azar.

$\boldsymbol{t} S$. quechuanum $=-0,287$, es decir, no existe correlación negativa entre la variación de la temperatura y los ejemplares colectados. Esa correlación se debe al azar.

\section{DISCUSIÓN}

El área de muestreo seleccionada mostró una marcada gradiente termal como lo plantean Vannote y Sweeney (1980), en las dos fechas de colecta tanto en la temperatura máxima como mínima.

Los estados inmaduros de S. escomeli mostraron una mayor abundancia en las colectas efectuadas en la sección donde debiera estar su óptimo ambiental.

\section{LITERATURA CITADA}

ADLER P.H. \& J.W. MC CREADIE. 1997. The hidden ecology of black flies: sibling species and ecological scale. The American Entomologist 43: 153-161.

CÉRÉGHINO R. \& P. LAVANDIER. 1997. Influence des éclusées hydroélectriques sur la distribution et le developpement larvaire des Diptères Simuliidae d'une rivière de moyenne. Cahiers de Recherche. Academie des Sciences, Paris, Sciences de la vie. 320: 329-338.

COSCARÓN S.Y.; C.L. COSCARÓN-ARIAS \& G. PORCARO. 2000. Simuliidae (Diptera) del Río Quequén Grande en las Cascadas (Buenos Aires, Argentina): Variación poblacional y relación con temperatura, caudal y precipitaciones. Acta Entomológica Chilena. 24: 29-36.
El análisis de correlación muestra que la mayor temperatura estaría influyendo en la abundancia de S. escomeli, especie hematófaga antropófila, lo que podría explicar las grandes poblaciones de esta especie. La presencia de las otras dos especies, $S$. philippii y $S$. quechuanum en la estación de muestreo donde se registraron temperaturas más bajas respecto a las otras dos estaciones, también permitiría explicar su presencia en sectores de mayor altitud en la región donde las temperaturas del agua son aún más bajas.

Un muestreo sistemático estacional debiera mostrar una migración río arriba y río abajo de S. escomeli, dependiendo de la temperatura media del río en el momento en que se realiza la colecta de sus estados inmaduros.

\section{CONCLUSIONES}

El área de estudio escogida presentó una gradiente termal durante el tiempo en que duró el estudio.

Se encontraron tres especies presentes durante el período de colecta en el área de estudio seleccionada: Simulium (Psilopelmia) escomeli, S. (Pternaspatha) philippii y S. (Pternaspatha) quechuanum.

S. escomeli fue la especie más abundante de las tres especies encontradas, presentando una correlación positiva intensa con la temperatura del agua; su coeficiente de determinación nos indica que en un 59,9\% que la variación en el número de ejemplares de esta especie se explica por la variación de la temperatura.

S. philippii y $S$. quechuanum presentaron una correlación negativa moderada y debil respectivamente con la temperatura del agua.

GIL M.; E.A. VALLANIA \& M.C. CORIGLIANO. 1998. Abundancia y distribución de Simuliidae (Diptera) en arroyos de las sierras de San Luis, Argentina. Revista Sociedad Entomológica Argentina. 57: 1-4.

HADI U.K. \& H. TAKAOKA. 1995. Effects of constant temperatures on oviposition and immature development of $S$. bidentatum (Diptera: Simuliidae) a vector of bovine Onchocerca (Nematoda: Onchoceridae) in Central Kyushu, Japan. Journal of Medical Entomology. 32: 801-806.

LAKE D.J. \& J. F. BURGER. 1983. Larval distribution and succession of outlet-breeding blackflies (Diptera: Simuliidae) in New Hampshire. Canadian Journal of Zoology 61: 2519-2533. 
MC CREADIE J.W. \& M.H. COLBO. 1991. Spatial distribution patterns of larval cytotypes of the Simulium venustum/verecundum complex (Diptera: Simuliidae) on the Avalon Peninsula, Newfoundland: factors associated with occurrence. Canadian Journal of Zoology. 69: $1389-1396$

MC CREADIE J.W. \& M.H. COLBO. 1992. Spatial distribution patterns of larval cytotypes of the Simulium venustum/ verecundum complex (Diptera: Simuliidae) on the Avalon Peninsula, Newfoundland: factors associated with cytotype abundance and composition. Canadian Journal of Zoology. 70: 1389-1396.

MC CREADIE J.W. \& P.H. ADLER. 1998. Scale, time, space and predictability: species distributions of preimaginal blackflies (Diptera: Simuliidae). Oecologia 114: 79-92.
NADEAU M.P.; G.B. DUNPHY \& J.L. BOISVERT. 1995.

Effects of physical factors on the development secondary conidia of Erynia conica (Zygomycetes: Entomophthorales) a pathogen of adult blackflies (Diptera: Simuliidae). Experimental Mycology. 19: 324-329.

ROSS D.H. \& R.W. MERRITT. 1987. Factors affecting larval blackfly distributions and population dynamic, pp. 90-108: In Blackflies ecology, population management and annotated list. K.C. Kim \& W. Merritt eds. Pennsylvania University State.

SPIEGEL M. R. 1997. Estadística. McGraw-Hill, $2^{\text {a }}$ Edición. $556 \mathrm{pp}$.

VANNOTE R.L. \& B.W.SWEENEY. 1980. Geographic analysis of thermal equilibria: a conceptual model for evaluating the effect of natural and modified thermal regimes on aquatic communities. American Naturalist. 115: 667-695. 